PROCEEDINGS OF THE

AMERICAN MATHEMATICAL SOCIETY

Volume 128, Number 2, Pages 561-572

S 0002-9939(99)05166-7

Article electronically published on July 6, 1999

\title{
THE ROLE OF BILLINGSLEY DIMENSIONS IN COMPUTING FRACTAL DIMENSIONS ON CANTOR-LIKE SPACES
}

\author{
JOSÉ-MANUEL REY \\ (Communicated by Frederick W. Gehring) \\ This paper is dedicated to Professor Juan Carlos Simó
}

\begin{abstract}
We consider a Cantor-like set as a geometric projection of a Bernoulli process. P. Billingsley (1960) and C. Dai and S.J. Taylor (1994) introduced dimension-like indices in the probability space of a stochastic process. Under suitable regularity conditions we find closed formulae linking the Hausdorff, box and packing metric dimensions of the subsets of the Cantor-like set, to the corresponding Billingsley dimensions associated with a suitable Gibbs measure. In particular, these formulae imply that computing dimensions in a number of well-known fractal spaces boils down to computing dimensions in the unit interval endowed with a suitable metric. We use these results to generalize density theorems in Cantor-like spaces. We also give some examples to illustrate the application of our results.
\end{abstract}

\section{INTRODUCTION}

In this note we take the standard viewpoint of thinking of Cantor-like constructions as geometric projections of the space of realizations of a Bernoulli process [11]. Billingsley [1] introduced a Hausdorff-like dimensional index in the probability space of a stochastic process to evaluate the size of null-probability events. Dai and Taylor [9] have recently defined, in a Billingsley setting, dimension indices inspired on the box and packing dimensions from geometric measure theory [20]. Since the dimension-like indices in [1,9] (Billingsley dimensions for the sequel) are defined as measure-theoretic objects, their analysis builds on the probability structure of the process. We investigate how fractal dimensions defined on different Cantor-like spaces can be obtained from Billingsley dimensions associated with the commom underlying process.

In section 2 we define a metric in the probability space of a Bernoulli process so that Billingsley dimensions are obtained as standard metric dimensions. To our knowledge, this fact has remained unnoticed in the literature $[1,2,6,9]$. In

Received by the editors October 28, 1997 and, in revised form, April 9, 1998.

1991 Mathematics Subject Classification. Primary 28A78, 28A80; Secondary 58F11.

This research was partially supported by a postdoctoral grant (A.P.E.) from the Universidad Complutense de Madrid. A preliminary version of this paper was written while visiting the Mathematical Institute at the University of St Andrews. The author thanks Kenneth J. Falconer and the members of the Analysis Research Group at St Andrews for their kind hospitality during his stay. Many useful discussions with Manuel Morán stimulated the initial development of this research. 
section 3 we obtain general formulae (see Theorem 3.6) linking Hausdorff, packing and box metric dimensions to the corresponding Billingsley dimensions defined in a suitable probability space, which is determined from the theory of Gibbs measures. Specifically, we prove that each fractal dimension of a subset of the space is a constant (the dimension of the space) times the corresponding Billingsley dimension of the associated subset of realizations of the process. We require some control on the geometry of the construction, and we show that a number of well-known fractal constructions are obtained as particular cases in this setting. The main result in this section can be seen as follows: once the ambient space $E$ has been constructed, fractal dimensions in $E$ can be computed in the unit interval endowed with an adequate metric, and thus the geometry of $E$ does not play any further role for computing dimensions in $E$. In section 4 we obtain density theorems which provide dimension bounds for the subsets of $E$ from information supplied by Billingsley dimensions. In section 5 we give some examples and applications of the results in earlier sections. In particular we apply our results to the multifractal description of some measures with Cantor-like support. The research described in this paper can be extended to constructions driven by more general (random) schemes.

\section{Preliminaries}

We first introduce the class of geometric constructions considered in this paper. Let $F \equiv F_{0}$ be a nonempty compact subset of $\mathbb{R}^{N}$. We assume that $|F|=1$, where $|\cdot|$ stands for diameter in $\mathbb{R}^{N}$. Let $m \geq 2$ be an integer, and $M=\{1,2, \ldots, m\}$. Let $\left\{F_{i_{1}, \ldots, i_{k}}: i_{j} \in M, j=1, \ldots, k, k \in \mathbb{N}\right\}$ be a collection of nonempty compact subsets of $F$ satisfying $F_{i_{1}, \ldots, i_{k}, j} \subset F_{i_{1}, \ldots, i_{k}}$ for $j \in M$ and $k \in \mathbb{N} \cup\{0\}$. We assume that $\max _{\left(i_{1}, \ldots, i_{k}\right)}\left|F_{i_{1}, \ldots, i_{k}}\right| \rightarrow 0$ as $k \rightarrow+\infty$. The Cantor-like set associated with the construction is the compact set defined by

$$
E=\bigcap_{k \in \mathbb{N}\left(i_{1}, \ldots, i_{k}\right) \in M^{k}} F_{i_{1}, \ldots, i_{k}} .
$$

The set $E$ can be considered as the image of the code space $M^{\infty}:=\chi_{1}^{\infty} M$ under the (coding) mapping $\pi: M^{\infty} \mapsto E$, defined by

$$
\pi\left(i_{1}, i_{2}, \ldots\right)=\bigcap_{k \in \mathbb{N}} F_{i_{1}, i_{2}, \ldots, i_{k}} .
$$

We next recall some standard definitions in geometric measure theory (see, e.g., $[10,13])$. Let $(X, d)$ be a metric space, $A \subseteq X$ and $\delta>0$. A collection $\mathcal{R}=\left\{B_{i}\right\}_{i \in \mathbb{N}}$ of closed balls in $X$ is a $\delta$-covering of $A$ if $A \subseteq \bigcup_{i} B_{i}$ and $\operatorname{diam}\left(B_{i}\right)<\delta$ for all $i$, where $\operatorname{diam}(\cdot)$ stands for the diameter of a subset in $(X, d)$. Assume that $B_{i}=B\left(x_{i}, \delta_{i}\right), \mathcal{R}$ is called a $\delta$-packing of $A$ if $x_{i} \in A, \delta_{i}<\delta$ for all $i$, and $B\left(x_{i}, \delta_{i}\right) \cap B\left(x_{j}, \delta_{j}\right)=\emptyset$ for $i \neq j$. Let $t \geq 0$. The $t$-dimensional (spherical) Hausdorff measure of $A$ is defined by

$$
H^{t}(A)=\sup _{\delta>0} \inf \left\{\sum_{i \in \mathbb{N}}\left(\operatorname{diam}\left(B_{i}\right)\right)^{t}:\left\{B_{i}\right\}_{i} \text { is a } \delta \text {-covering of } A\right\} .
$$

The Hausdorff dimension of $A$ can be defined by $\operatorname{dim} A=\inf \left\{t: H^{t}(A)=0\right\}$. The $t$-dimensional packing pre-measure of $A$ is defined by

$$
\bar{P}^{t}(A)=\inf _{\delta>0} \sup \left\{\sum_{i \in \mathbb{N}}\left(2 \delta_{i}\right)^{t}:\left\{B\left(x_{i}, \delta_{i}\right)\right\}_{i} \text { is a } \delta \text {-packing of } A\right\} .
$$


The (upper) box or Minkowski dimension of $A$ is given by $\Delta(A)=\inf \left\{t: \bar{P}^{t}(A)=\right.$ $0\}$. The $t$-dimensional packing measure of $A$ is defined by

$$
P^{t}(A)=\inf \left\{\sum_{i \in \mathbb{N}} \bar{P}^{t}\left(A_{i}\right): \bigcup_{i \in \mathbb{N}} A_{i} \supseteq A, A_{i} \subset X\right\},
$$

and the packing dimension of $A$ is the value given by $\operatorname{Dim} A=\inf \left\{t: P^{t}(A)=0\right\}$. It is well known that $\operatorname{dim}(A) \leq \operatorname{Dim}(A) \leq \Delta(A)$ for $A \subseteq X$.

Let $M^{*}=\bigcup_{k \in \mathbb{N}} M^{k}$. Given $\mathbf{i}=\left(i_{1}, i_{2}, \ldots\right) \in M^{\infty}$ and $k \in \mathbb{N}, \mathbf{i}(k)$ stands for the sequence $\left(i_{1}, i_{2}, \ldots, i_{k}\right)$. For $\mathbf{j} \in M^{k}$ we will write $l(\mathbf{j})=k$; the set $[\mathbf{j}]:=\left\{\mathbf{i} \in M^{\infty}\right.$ : $\mathbf{i}(k)=\mathbf{j}\}$ is called a cylinder set. The $\sigma$-algebra generated by the cylinder sets will be denoted by $\sigma(\mathcal{C})$. Let $\nu$ be a probability measure defined on $\left(M^{\infty}, \sigma(\mathcal{C})\right)$. We define, for $\mathbf{i}, \mathbf{j} \in M^{\infty}$,

$$
d_{\nu}(\mathbf{i}, \mathbf{j})= \begin{cases}\nu([\mathbf{i} \wedge \mathbf{j}]), & \mathbf{i} \neq \mathbf{j} \\ 0, & \mathbf{i}=\mathbf{j}\end{cases}
$$

where $\mathbf{i} \wedge \mathbf{j}$ is the longest finite sequence heading both $\mathbf{i}$ and $\mathbf{j}$, i.e. if $k=l(\mathbf{i} \wedge \mathbf{j}) \geq 0$, then $\mathbf{i}(k)=\mathbf{j}(k)=\mathbf{i} \wedge \mathbf{j}$ but $i_{k+1} \neq j_{k+1}$.

In the following lemma we assume that every cylinder has positive $\nu$-measure. This is a strong assumption but it is not necessary to obtain Billingsley dimensions as standard metric dimensions in the general case (see Remark 2.3 below).

Lemma 2.1. Let $\nu$ be a non-atomic probability measure on $\left(M^{\infty}, \sigma(\mathcal{C})\right)$ such that $\nu([\mathbf{i}])>0$ for all $\mathbf{i} \in M^{*}$; then $\left(M^{\infty}, d_{\nu}\right)$ is a compact, complete, and separable metric space.

Proof. To check the triangle inequality

$$
d_{\nu}(\mathbf{i}, \mathbf{j}) \leq d_{\nu}(\mathbf{i}, \mathbf{k})+d_{\nu}(\mathbf{k}, \mathbf{j}),
$$

take three distinct codes $\mathbf{i}, \mathbf{j}, \mathbf{k} \in M^{\infty}$ (otherwise (2.4) is trivial) and let $l(\mathbf{i} \wedge \mathbf{j})=n$. In case that $n \geq 1$ and $0 \leq l(\mathbf{k} \wedge \mathbf{i})<n$, then $[\mathbf{i} \wedge \mathbf{j}] \subset[\mathbf{k} \wedge \mathbf{i}]$. If $l(\mathbf{k} \wedge \mathbf{i}) \geq n \geq 0$, then $\min \{l(\mathbf{k} \wedge \mathbf{i}), l(\mathbf{k} \wedge \mathbf{j})\}=n$ (otherwise $l(\mathbf{i} \wedge \mathbf{j})>n)$ and thus either $[\mathbf{i} \wedge \mathbf{j}]=[\mathbf{k} \wedge \mathbf{j}]$ or $[\mathbf{i} \wedge \mathbf{j}]=[\mathbf{k} \wedge \mathbf{i}]$. In any case (2.4) follows from (2.3). Since the metric $d_{\nu}$ induces the product topology, the result follows.

Since $\operatorname{diam}([\mathbf{i}])=\nu([\mathbf{i}])$ for all $\mathbf{i} \in M^{*}$, Lemma 2.1 implies that the definition of Hausdorff dimension in the metric space $\left(M^{\infty}, d_{\nu}\right)$ coincides with the dimension defined by Billingsley in the probability space $\left(M^{\infty}, \sigma(\mathcal{C}), \nu\right)$ [1]. Similarly, the definitions of box and packing dimensions in $\left(M^{\infty}, d_{\nu}\right)$ coincide, respectively, with the definitions of box and packing dimensions in $\left(M^{\infty}, \sigma(\mathcal{C}), \nu\right)$ given by Dai and Taylor in [9]. In view of Lemma 2.1, dimension-like properties of Billingsley dimensions, say $\sigma$-stability, are consequences of the theory of dimension in metric spaces (see, e.g., [8]). Following $[1,9]$, we will call a $\delta$-covering (respectively, a $\delta$-packing) in $\left(M^{\infty}, d_{\nu}\right)$ a $(\nu, \delta)$-covering (resp. a $(\nu, \delta)$-packing); and we will denote the Hausdorff, box, and packing Billingsley dimensions associated with $\nu$ by $\operatorname{dim}_{\nu}, \Delta_{\nu}$ and $\operatorname{Dim}_{\nu}$ respectively. We will use below the fact that $\operatorname{dim}_{\nu}(A)=1$ if $\nu(A)>0$.

Remark 2.2. Let $(\Omega, \mathcal{A}, \nu)$ be a non-atomic probability space in which a stochastic process with discrete state space is defined. A metric as $d_{\nu}$ in (2.3) can be defined in $\Omega$ provided that the $\sigma$-algebra $\mathcal{A}$ contains the class of cylinder sets, and that every cylinder set has positive $\nu$-measure. Therefore Billingsley dimensions are obtained from classical definitions of metric dimensions also in this general case. 
Remark 2.3. Notice that Billingsley dimensions are obtained as metric dimensions also in the case when some cylinder has null $\nu$-measure. This is a consequence of the definitions of Billingsley dimensions and of the metric $d_{\nu}$ above. In such a case, $d_{\nu}$ is not a "nice" metric but rather a pseudometric in the code space $M^{\infty}$. We have required that $\nu([\mathbf{i}])>0$ for all $\mathbf{i} \in M^{*}$ in Lemma 2.1 since this holds for the Gibbs measure we consider here (see Remark 3.1).

\section{Linking GeOMEtric Dimensions to BiLlingsley's Dimensions}

Let $\mathcal{H}$ denote the class of Hölder-continuous functions $\phi: M^{\infty} \mapsto(0,+\infty)$, and let $\Pi(\phi)$ denote the topological pressure of $\phi$, that is,

$$
\Pi(\phi)=\lim _{n \rightarrow+\infty} \frac{1}{n} \log \sum_{\mathbf{i}^{\prime} \in M^{n}} \exp \left(\sup _{\mathbf{i} \in\left[\mathbf{i}^{\prime}\right] \cap M^{\infty}} S_{n} \phi(\mathbf{i})\right),
$$

where $S_{n} \phi(\mathbf{i}):=\sum_{k=0}^{n-1} \phi\left(\tau^{k} \mathbf{i}\right)$ and $\tau\left(i_{1}, i_{2}, \ldots\right)=\left(i_{2}, i_{3}, \ldots\right)$ is the Bernoulli shift on $M^{\infty}$. It is well known that there is a unique real number $s=s_{\phi} \geq 0$ such that

$$
\Pi(-s \phi)=0 .
$$

Furthermore, there exists a unique Borel probability measure $\nu_{\phi}$ such that, for some $\zeta>0$ and for any $\mathbf{i} \in M^{\infty}$ and $n \in \mathbb{N}$,

$$
\zeta^{-1} \exp \left(-s_{\phi} S_{n} \phi(\mathbf{i})\right)<\nu_{\phi}([\mathbf{i}(n)])<\zeta \exp \left(-s_{\phi} S_{n} \phi(\mathbf{i})\right) .
$$

We will call $\nu_{\phi}$ the Gibbs measure associated with $\phi$. See [5] for the theory of Gibbs measures and the thermodynamic formalism.

Remark 3.1. Since $\phi \in \mathcal{H}$ is bounded away from zero, (3.2) implies that the measure $\nu_{\phi}$ satisfies the hypotheses of Lemma 2.1 .

We will impose some conditions on the construction, similar to those defined by Pesin and Weiss in [18]. We say that $\phi \in \mathcal{H}$ is $u$-estimating for the Cantor-like construction defined by (2.1) if there exists $C_{u}>0$ such that, for all $\mathbf{i} \in M^{\infty}$ and $n \in \mathbb{N}$,

$$
\left|F_{\mathbf{i}(n)}\right| \leq C_{u} \exp \left(-S_{n} \phi(\mathbf{i})\right) .
$$

This is a strong uniform condition on the size of the sets $F_{\mathbf{i}(n)}$, which is satisfied by a number of classical constructions (see Examples 3.7 and 3.8 below).

Lemma 3.2. Let $\phi$ be u-estimating for the construction (2.1), and let $\nu_{\phi}$ be the Gibbs measure associated with $\phi$. For any $A \subseteq M^{\infty}$, $\operatorname{dim} \pi(A) \leq s_{\phi} \operatorname{dim}_{\nu_{\phi}}(A)$, $\Delta \pi(A) \leq s_{\phi} \Delta_{\nu_{\phi}}(A), \operatorname{Dim} \pi(A) \leq s_{\phi} \operatorname{Dim}_{\nu_{\phi}}(A)$, where $\pi$ is defined in (2.2) and $s_{\phi}$ is given by (3.1).

Proof. Endow $M^{\infty}$ with the metric $d_{\phi}:=d_{\nu_{\phi}}$ defined in (2.3). Since $\phi$ is $u^{-}$ estimating it follows from (2.2), (3.2), and (2.3) that, for $\mathbf{i}, \mathbf{j} \in M^{\infty}$,

$$
\operatorname{dist}(\pi(\mathbf{i}), \pi(\mathbf{j})) \leq\left|F_{\mathbf{i} \wedge \mathbf{j}}\right| \leq C_{u} \exp \left(-S_{l(\mathbf{i} \wedge \mathbf{j})} \phi(\mathbf{i})\right)<C_{u} \zeta^{1 / s_{\phi}}\left(d_{\phi}(\mathbf{i}, \mathbf{j})\right)^{1 / s_{\phi}}
$$

(dist denotes the euclidean metric). From [10, Proposition 2.3] and Lemma 2.1, it follows that $\operatorname{dim} \pi(A) \leq s_{\phi} d_{\phi}-\operatorname{dim}(A)=s_{\phi} \operatorname{dim}_{\nu_{\phi}} A$, where $d_{\phi}$ - $\operatorname{dim}$ denotes Hausdorff dimension computed in the metric space $\left(M^{\infty}, d_{\phi}\right)$. Notice that the proof of [10, Proposition 2.3] applies in our case. The inequalities for the box and packing dimensions follow in a similar way. 
Let $\phi \in \mathcal{H}$. Recall that $l(\mathbf{i})=k$ whenever $\mathbf{i} \in M^{k}$. For $\mathbf{i} \in M^{*}$, let $\gamma_{\mathbf{i}}:=$ $\sup _{\mathbf{i}^{\prime} \in[\mathbf{i}]}\left\{\exp \left(-S_{l(\mathbf{i})} \phi\left(\mathbf{i}^{\prime}\right)\right)\right\}$. Given a ball $B$ of small radius $r>0$, we define

$$
\mathcal{G}_{\phi}(B)=\left\{\mathbf{i} \in M^{*}: \gamma_{\mathbf{i}}<r \leq \gamma_{\mathbf{i}(l(\mathbf{i})-1)}, \pi([\mathbf{i}]) \cap B \neq \emptyset\right\} .
$$

We say that $\phi$ is $l$-estimating for the construction (2.1) if there exists $q=q_{\phi}<+\infty$ (independent of $r$ ) such that $\operatorname{card} \mathcal{G}_{\phi}(B)<q$ for any ball $B$ of radius $r$. Whenever such a $\phi$ exists the class of cylinders in (3.3) can be used instead of balls to obtain lower bounds for the fractal dimensions of subsets of the Cantor-like set $E$.

Lemma 3.3. Let $\phi \in \mathcal{H}$ be l-estimating for the construction (2.1), and let $\nu_{\phi}$ be the associated Gibbs measure. For any $A \subseteq M^{\infty}, \operatorname{dim} \pi(A) \geq s_{\phi} \operatorname{dim}_{\nu_{\phi}}(A)$.

Proof. Let $t<\operatorname{dim}_{\nu_{\phi}} A$, and let $\delta_{0}>0$ be such that

$$
\sum_{i \in \mathbb{N}} \nu_{\phi}\left(C_{i}\right)^{t}>0
$$

for any $\left(\nu_{\phi}, \delta_{0}\right)$-covering $\left\{C_{i}\right\}_{i}$ of $A$ by cylinders. Write $s=s_{\phi}$ and let $\delta<$ $2\left(\zeta^{-1} \delta_{0}\right)^{1 / s}$, where $\zeta$ is the constant in (3.2). Let $\left\{B_{i}\right\}_{i}$ be a $\delta$-covering of $\pi(A)$ by balls. From (3.3) and (3.2), it follows that $\bigcup_{i}\left\{[\mathbf{i}]: \mathbf{i} \in \mathcal{G}_{\phi}\left(B_{i}\right)\right\}$ is a $\left(\nu_{\phi}, \delta_{0}\right)$-covering of $\pi^{-1}(\pi(A)) \supseteq A$. Since $\phi$ is $l$-estimating, (3.2) and (3.4) together give

$$
\sum_{i}\left|B_{i}\right|^{s t}>2^{s t} q^{-1} \sum_{i} \sum_{\mathbf{i} \in \mathcal{G}_{\phi}\left(B_{i}\right)} \gamma_{\mathbf{i}}^{s t}>2^{s t} q^{-1} \zeta^{-t} \sum_{i} \sum_{\mathbf{i} \in \mathcal{G}_{\phi}\left(B_{i}\right)} \nu_{\phi}([\mathbf{i}])^{t}>0,
$$

and therefore $\operatorname{dim} \pi(A) \geq s t$.

For a subset $A$ of a metric space and $\delta>0$, let $N(A, \delta)$ denote the minimal number of closed balls of diameter $\delta$ (or less) which cover $A$; the box dimension satisfies (see [8])

$$
\Delta(A)=\limsup _{\delta \rightarrow 0} \frac{\log N(A, \delta)}{-\log \delta} .
$$

Lemma 3.4. Let $\phi \in \mathcal{H}$ be l-estimating for the construction (2.1), and let $\nu_{\phi}$ be the associated Gibbs measure. For any $A \subseteq M^{\infty}$ such that $\Delta_{\nu_{\phi}}(A) \geq \beta$, we have $\Delta \pi(A) \geq s_{\phi} \beta$.

Proof. Write $s=s_{\phi}$ and assume that $\Delta \pi(A)<s \beta$. It follows that for all $\delta>0$ small enough, $N_{\delta}^{*}<\delta^{-s \beta}$, where $N_{\delta}^{*}$ denotes the minimum number of closed balls with radii $\delta$ that cover $\pi(A)$ [10]. Let $B_{1}, \ldots, B_{N_{\delta}^{*}}$ be $N_{\delta}^{*}$ closed balls of radii $\delta$ covering $\pi(A)$, and consider the collection of cylinders $\mathcal{G}(\delta):=\bigcup_{i=1}^{N_{\delta}^{*}}\left\{[\mathbf{i}]: \mathbf{i} \in \mathcal{G}_{\phi}\left(B_{i}\right)\right\}$. From $(2.3),(3.2)$, and (3.3) it follows that $\mathcal{G}(\delta)$ is a $\left(\nu_{\phi}, \zeta \delta^{s}\right)$-covering of $A$. Since $\phi$ is $l$-estimating, $\operatorname{card} \mathcal{G}(\delta)<q N_{\delta}^{*}$, and thus

$$
\limsup _{\delta \rightarrow 0} \frac{\log N\left(A, \zeta \delta^{s}\right)}{-\log \left(\zeta \delta^{s}\right)}<\limsup _{\delta \rightarrow 0} \frac{\log \left(q N_{\delta}^{*}\right)}{-\log \left(\zeta \delta^{s}\right)}<\beta .
$$

Definition (3.5), Remark 3.1 and Lemma 2.1 together imply that $\Delta_{\nu_{\phi}}(A)<\beta$.

For any subset $A$ of a metric space, it is proved in [8] that

$$
\operatorname{Dim} A=\inf \left\{\sup _{i \in \mathbb{N}} \Delta\left(A_{i}\right): A_{i} \uparrow A\right\} .
$$

Lemma 3.5. Let $\phi \in \mathcal{H}$ be l-estimating for the construction (2.1), and let $\nu_{\phi}$ be the Gibbs measure associated with $\phi$. For $A \subseteq M^{\infty}, \operatorname{Dim} \pi(A) \geq s_{\phi} \operatorname{Dim}_{\nu_{\phi}}(A)$. 
Proof. Assume that $\operatorname{Dim} \pi(A)<s_{\phi} \beta$. From (3.6) there is $\left\{A_{i}\right\}_{i}$ such that $A_{i} \uparrow \pi(A)$ and $\Delta A_{i}<s_{\phi} \beta$ for all $i$. Lemma 3.4 gives $\Delta_{\nu_{\phi}}\left(\pi^{-1}\left(A_{i}\right) \cap A\right)<\beta$ for all $i$. Since $\left(\pi^{-1}\left(A_{i}\right) \cap A\right) \uparrow A,(3.6)$ and Lemma 2.1 give $\operatorname{Dim}_{\nu_{\phi}}(A)<\beta$.

We say that the Cantor-like construction (2.1) is $\phi$-estimated if there exists a function $\phi \in \mathcal{H}$ which is both $u$-estimating and $l$-estimating for the construction. The condition that the construction is $\phi$-estimated allows us to compute geometric dimensions within the space $E$ using cylinders sets in the metric space $\left(M^{\infty}, d_{\phi}\right)$.

Theorem 3.6. Let $E$ be the limit set of a $\phi$-estimated Cantor-like construction, and let $\nu_{\phi}$ be the associated Gibbs measure. For any $A \subseteq M^{\infty}$ it holds that $\operatorname{dim} \pi(A)=s_{\phi} \operatorname{dim}_{\nu_{\phi}}(A), \Delta \pi(A)=s_{\phi} \Delta_{\nu_{\phi}}(A)$, and $\operatorname{Dim} \pi(A)=s_{\phi} \operatorname{Dim}_{\nu_{\phi}}(A)$, where $\pi$ is given by (2.2) and $s_{\phi}$ is defined by (3.1).

Notice that Theorem 3.6 reduces different geometric-size problems (e.g., consider different $\phi$-estimated spaces) to the same question on $M^{\infty}$. This is a sort of rigidity property, independent of the geometry of the ambient space. Since $M^{\infty}$ is identified with the unit interval except for a countable set of codes, Lemma 2.1 and Theorem 3.6 imply that computing dimensions in $\phi$-estimated spaces boils down to computing dimensions in $\left([0,1], d_{\phi}\right)$. We collect below some well-known geometric constructions that are $\phi$-estimated.

Example 3.7. Moran-like constructions [18]. The basic sets $\left\{F_{\mathbf{i}}: \mathbf{i} \in M^{*}\right\}$ satisfy: i) there exist $0<r_{i}<1, i \in M$, and positive constants $C_{1}, C_{2}$ such that, for all $\mathbf{i} \in M^{*}, \underline{B}_{\mathbf{i}} \subset F_{\mathbf{i}} \subset \bar{B}_{\mathbf{i}}$, where $\underline{B}_{\mathbf{i}}$ and $\bar{B}_{\mathbf{i}}$ are closed balls with radii $C_{1} r_{\mathbf{i}}$ and $C_{2} r_{\mathbf{i}}$, respectively (we abbreviate the product $r_{i_{1}} \cdots r_{i_{l(\mathrm{i})}}$ by $r_{\mathbf{i}}$ ).

ii) $\operatorname{int} \underline{B}_{\mathbf{i}} \cap \operatorname{int} \underline{B}_{\mathbf{j}}=\emptyset$ for all $\mathbf{i}, \mathbf{j} \in M^{*}$ such that neither $[\mathbf{i}] \subset[\mathbf{j}]$ nor $[\mathbf{i}] \supset[\mathbf{j}]$.

This type of constructions includes as a particular case the classical Moran construction in which $F_{i_{1}, \ldots, i_{k}, j}$ is geometrically similar to $F_{i_{1}, \ldots, i_{k}}$ with similarity ratio given by $r_{j}$. The construction is $\phi$-estimated by $\phi(\mathbf{i})=-\log r_{i_{1}}$ (that $\phi$ is $l$-estimating follows from [11, Lemma 5.3.1]).

Example 3.8. Map-driven constructions. The construction is driven by maps if there exists a set of contractive mappings $\Psi=\left\{\varphi_{i}: i \in M\right\}$ such that $F_{\mathbf{i}}=\varphi_{\mathbf{i}}(F)$ for all $\mathbf{i} \in M^{*}$, where $\varphi_{\mathbf{i}}:=\varphi_{i_{1}} \circ \varphi_{i_{2}} \circ \cdots \circ \varphi_{i_{l(\mathbf{i})}}$. If the mappings $\varphi_{i}$ are conformal $C^{1+\eta}$-diffeomorphisms, $\eta>0$, defined on some open set $U \supseteq F ; E$ is called a self-conformal set [3]. If the construction takes place in $\mathbb{R}$ and a strong separation condition holds, E is called a cookie-cutter set [10]. A Cantor-like construction is said to satisfy the open set condition if

$$
\operatorname{int} F_{\mathbf{i}} \cap \operatorname{int} F_{\mathbf{j}}=\emptyset, \text { for all } \mathbf{i}, \mathbf{j} \in M^{n}, \mathbf{i} \neq \mathbf{j}, n \in \mathbb{N} .
$$

Self-conformal constructions satisfying (3.7) are $\phi$-estimated by

$$
\phi(\mathbf{i})=-\log \left|\varphi_{i_{1}}^{\prime}(\pi(\tau(\mathbf{i})))\right| .
$$

This fact follows from results in [3]. If the mappings $\varphi_{j}$ are similarities, the set $E$ is called self-similar. If condition (3.7) holds, the construction is $\phi$-estimated by $\phi(\mathbf{i})=-\log r_{i_{1}}$, where here $r_{j}:=\operatorname{lip} \varphi_{j}$ is the contraction ratio of $\varphi_{j}$. The dimensions of $E$ are given by the 'similarity dimension' $s$ of $\Psi$, defined by

$$
\sum_{j \in M} r_{j}^{s}=1
$$

(see $[11,10]$ ), which is a particular case of the pressure equation (3.1). 
Remark 3.9. The unit interval is the self-similar set generated by the similarities $\varphi_{i}(x)=m^{-1}(x+i), i \in\{0,1, \ldots, m-1\}$. We thus get the so-called 'Lebesgue case' considered in $[1,9]$, where it was shown that the Hausdorff and packing Billingsley dimensions associated with $\nu:=\times_{1}^{\infty}\left(\frac{1}{m},(m), \frac{1}{m}\right)$ coincide, respectively, with the Hausdorff and packing metric dimensions in the real line. Theorem 3.6 thus generalizes these facts to the case of Cantor-like constructions.

\section{Generalizing Density theorems in CANTOR-Like CONSTRUCtions}

A standard approach to obtain lower bounds for the dimensions of a set $B \subset \mathbb{R}^{N}$ is to distribute a probability measure $\mu$ so that $\mu(B)>0$ and a suitable local density of $\mu$ (see, e.g., (5.8) and (5.9) below) is uniformly bounded on $B[10,13,20,9,15]$. Theorem 4.1 below weakens the hypotheses of standard density theorems in the sense that the set $B$ may not have positive $\mu$-measure. If $B$ is a $\mu$-null subset of a Cantor-like space, information about its $\mu$-size supplied by Billingsley dimension still allows us to obtain bounds for its Hausdorff dimension.

Theorem 4.1. Let $\phi$ be an l-estimating function for the Cantor-like construction (2.1), and let $\nu_{\phi}$ be the associated Gibbs measure. Let $A \subseteq M^{\infty}$, assume that there is a probability measure $\nu$ such that $\liminf _{k \rightarrow+\infty} \frac{\log \nu([\mathbf{i}(k)])}{\log \nu_{\phi}([\mathbf{i}(k)])} \geq \beta$ for all $\mathbf{i} \in A$, and assume that $\operatorname{dim}_{\nu}(A) \geq \gamma$. Then $\operatorname{dim} \pi(A) \geq s_{\phi} \beta \gamma$, where $s_{\phi}$ is given by (3.1).

Proof. From [2, Theorem 2.2] $\operatorname{dim}_{\nu_{\phi}}(A) \geq \beta \gamma$ so that Lemma 3.3 concludes.

To prove a packing version of Theorem 4.1 we need the following lemma.

Lemma 4.2. Let $\nu$ and $\mu$ be two probability measures defined in $\left(M^{\infty}, \sigma(\mathcal{C})\right), \nu$ non-atomic, and let $A \subseteq M^{\infty}$ such that $\limsup _{k \rightarrow+\infty} \frac{\log \nu([\mathbf{i}(k)])}{\log \mu([\mathbf{i}(k)])} \leq \beta$ for all $\mathbf{i} \in A$; then $\operatorname{Dim}_{\mu} A \leq \beta \operatorname{Dim}_{\nu} A$.

Proof. Let $t>\operatorname{Dim}_{\nu} A$. From [9, Theorem 4.1(vi)] there exists $A_{j} \uparrow A$ such that $\lim _{j \rightarrow+\infty} \bar{p}_{\nu}^{t}\left(A_{j}\right)<1$. Let $j$ be large enough, and let $\delta_{0}=\delta_{0}(j)>0$ be such that

$$
\sum_{C \in \Gamma} \nu(C)^{t}<1
$$

for any $\left(\nu, \delta_{0}\right)$-packing $\Gamma$ of $A_{j}$. Let $\xi>\beta$, and take $n \geq \min \left\{q \in \mathbb{N}: \max _{\mathbf{i} \in M^{q}} \nu([\mathbf{i}])\right.$ $\left.<\delta_{0}\right\}$. Define

$$
A_{j, n}=\bigcap_{k>n}\left\{\mathbf{i} \in A_{j}: \nu([\mathbf{i}(k)])>\mu([\mathbf{i}(k)])^{\xi}\right\} .
$$

Let $\delta<\min \left\{\mu([\mathbf{i}]): \mu([\mathbf{i}])>0, \mathbf{i} \in M^{n}\right\}$, and let $\left\{C_{i}\right\}_{i \in \mathbb{N}}$ be a $(\mu, \delta)$-packing of $A_{j, n}$. Notice that $C_{i}=[\mathbf{i}(k)]$ for some $k>n$ and for some $\mathbf{i} \in A_{j, n}$, so that $\nu\left(C_{i}\right)<\delta_{0}$. Since $\left\{C_{i}\right\}_{i}$ is also a $\left(\nu, \delta_{0}\right)$-packing of $A_{j, n},(4.1)$ and (4.2) together give

$$
\sum_{i} \mu\left(C_{i}\right)^{\xi t}<\sum_{i} \nu\left(C_{i}\right)^{t}<1,
$$

and therefore $\bar{p}_{\mu}^{\xi t}\left(A_{j, n}\right)<1$. Since $A_{j, n} \uparrow A_{j}$ as $n \rightarrow+\infty$, Theorem 4.1(vi) in [9] implies that

$$
p_{\mu}^{\xi t}\left(A_{j}\right) \leq \lim _{n \rightarrow+\infty} \bar{p}_{\mu}^{\xi t}\left(A_{j, n}\right) \leq 1
$$


Since $A_{j} \uparrow A$, $[9$, Theorem $4.1(\mathrm{v})]$ gives $p_{\mu}^{\xi t}(A)=\lim _{j \rightarrow+\infty} p_{\mu}^{\xi t}\left(A_{j}\right) \leq 1$ so that $\operatorname{Dim}_{\mu}(A) \leq \xi t$.

Theorem 4.3 below provides upper bounds for packing dimensions which are sharper than those obtained from standard density theorems $[13,8,15]$ in the case that $\gamma<1$. The proof follows from Lemmas 4.2 and 3.2.

Theorem 4.3. Let $\phi$ be u-estimating for the construction (2.1), and let $\nu_{\phi}$ be the associated Gibbs measure. Let $A \subseteq M^{\infty}$, assume that there is a non-atomic probability measure $\nu$ such that $\limsup _{k \rightarrow+\infty} \frac{\log \nu([\mathbf{i}(k)])}{\log \nu_{\phi}([\mathbf{i}(k)])} \leq \beta$ for all $\mathbf{i} \in A$, and assume that $\operatorname{Dim}_{\nu} A \leq \gamma$. Then $\operatorname{Dim} \pi(A) \leq s_{\phi} \beta \gamma$, where $s_{\phi}$ is given by (3.1).

\section{Some APplications}

5.1. Multifractal analysis of measures supported on Cantor-like spaces. The results in previous sections can be applied to the computation of the multifractal spectra of a class of measures defined on $\phi$-estimated Cantor-like spaces. Relevant papers on multifractality include $[4,7,16]$. If $\nu$ is a probability measure defined in the metric space $\left(M^{\infty}, d_{\phi}\right)$ and $\mu:=\nu \circ \pi^{-1}$ is the induced measure supported on a $\phi$-estimated Cantor-like space $E$, the (spherical) multifractal component of level $\alpha \geq 0$ of $\mu$ is the set defined as

$$
E(\alpha)=\left\{\pi(\mathbf{i}) \in E: \alpha_{\mu}(\pi(\mathbf{i})):=\lim _{r \rightarrow 0} \frac{\log \mu(B(\pi(\mathbf{i}), r))}{\log r}=\alpha\right\} .
$$

The Hausdorff and packing multifractal spectra of $\mu$ are, respectively, the functions

$$
\alpha \mapsto f_{\mu}(\alpha):=\operatorname{dim} E(\alpha), \quad \alpha \mapsto F_{\mu}(\alpha):=\operatorname{Dim} E(\alpha) .
$$

The cylindrical multifractal spectra of the measure $\mu$ are defined as above but in terms of the cylindrical logarithmic density, that is,

$$
\alpha \mapsto \operatorname{dim} C(\alpha), \quad \alpha \mapsto \operatorname{Dim} C(\alpha),
$$

where

$$
C(\alpha)=\left\{\pi(\mathbf{i}): \lim _{n \rightarrow+\infty} \frac{\log \mu\left(F_{\mathbf{i}(n)}\right)}{\log \left|F_{\mathbf{i}(n)}\right|}=\alpha\right\} .
$$

It has been proven that the spherical and the cylindrical multifractal spectra coincide in several important cases, namely for product measures on self-similar totally disconnected constructions [7], then for product measures on self-conformal (and thus self-similar) constructions satisfying the strong open set condition [17], and for equilibrium measures on disjointed Moran-like constructions [19] (see Examples 3.7 and 3.8). Since all these constructions are $\phi$-estimated, it follows from the results in section 3 that, for all those cases,

$$
f_{\mu}(\alpha)=\operatorname{dim} C(\alpha)=s_{\phi} \mathfrak{f}_{\nu}\left(\frac{\alpha}{s_{\phi}}\right), \quad F_{\mu}(\alpha)=\operatorname{Dim} C(\alpha)=s_{\phi} \mathfrak{F}_{\nu}\left(\frac{\alpha}{s_{\phi}}\right),
$$

where $\mathfrak{f}_{\nu}(\cdot)$ and $\mathfrak{F}_{\nu}(\cdot)$ denote, respectively, the Hausdorff and packing multifractal spectra of the measure $\nu$ computed in the space $\left(M^{\infty}, d_{\phi}\right)$. In view of (5.1), the computations of a number of classical multifractal spectra boil down to the computations of the multifractal spectra of the inducing measure on the code space 
endowed with the metric $d_{\phi}$. Moreover, the formulae in (5.1) suggest that whenever the multifractal formalism (see e.g. [16]) holds for the geometric measure $\mu$, it also holds for the inducing measure $\nu$ and vice versa.

5.2. Dimension bounds for Besicovitch frequential sets. Given $\mathbf{i} \in M^{\infty}$, $\mathbf{j} \in M^{*}$, and $n \geq l(\mathbf{j})$, let

$$
\delta_{\mathbf{j}}(\mathbf{i}, n)=\frac{1}{n} \operatorname{card}\left\{q: i_{q}=j_{1}, i_{q+1}=j_{2}, \ldots, i_{q+l(\mathbf{j})-1}=j_{l(\mathbf{j})}, 1 \leq q \leq n-l(\mathbf{j})+1\right\} ;
$$

and write $\delta_{\mathbf{j}}(\mathbf{i})=\lim _{n \rightarrow+\infty} \delta_{\mathbf{j}}(\mathbf{i}, n)$ whenever such a limit exists.

Let $\mathcal{P}^{+}:=\left\{\left(\xi_{j}\right)_{j \in M}: \xi_{j}>0, j \in M, \sum_{j \in M} \xi_{j}=1\right\}$; and define, for $\mathrm{p}=$ $\left(p_{j}\right)_{j \in M} \in \mathcal{P}^{+}$,

$$
B_{\mathrm{p}}=\bigcap_{j \in M}\left\{\mathbf{i} \in M^{\infty}: \delta_{j}(\mathbf{i})=p_{j}\right\},
$$

where $\delta_{j}(\mathbf{i})=\lim _{n \rightarrow+\infty} \delta_{j}(\mathbf{i}, n)$. Let $E$ be the limit set defined in (2.1) and let $\pi$ be the associated map defined in (2.2). We call the set $\pi\left(B_{\mathrm{p}}\right)$ the Besicovitch normal set associated with $(\pi, \mathrm{p})$. Let $0<\underline{r}_{j} \leq \bar{r}_{j}<1$ for $j \in M$, and assume that $\bar{\phi}(\mathbf{i})=-\log \bar{r}_{i_{1}}$ and $\underline{\phi}(\mathbf{i})=-\log \underline{r}_{i_{1}}$ are $u$-estimating and $l$-estimating for the construction, respectively. Consider the product measure $\nu_{\mathrm{p}}:=\chi_{1}^{\infty} \mathrm{p}$. If $\nu_{\bar{\phi}}$ denotes the Gibbs measure associated with $\bar{\phi},(3.2)$ and (5.2) together give

$$
\limsup _{k \rightarrow+\infty} \frac{\log \nu_{\mathrm{p}}([\mathbf{i}(k)])}{\log \nu_{\bar{\phi}}([\mathbf{i}(k)])} \leq \frac{\sum_{j \in M} p_{j} \log p_{j}}{s_{\bar{\phi}} \sum_{j \in M} p_{j} \log \bar{r}_{j}}:=\bar{\beta}(\mathrm{p}),
$$

for all $\mathbf{i} \in B_{\mathrm{p}}$. From the strong law of large numbers, $\nu_{\mathrm{p}}\left(B_{\mathrm{p}}\right)=1$ and thus $\operatorname{dim}_{\nu_{\mathrm{p}}} B_{\mathrm{p}}=\operatorname{Dim}_{\nu_{\mathrm{p}}} B_{\mathrm{p}}=1$, so that Theorem 4.3 gives $\operatorname{Dim} \pi\left(B_{\mathrm{p}}\right) \leq s_{\bar{\phi}} \bar{\beta}(\mathrm{p})$. Since

$$
\underline{\beta}(\mathrm{p}):=\frac{\sum_{j \in M} p_{j} \log p_{j}}{s_{\underline{\phi}} \sum_{j \in M} p_{j} \log \underline{r}_{j}} \leq \liminf _{k \rightarrow+\infty} \frac{\log \nu_{\mathrm{p}}([\mathbf{i}(k)])}{\log \nu_{\underline{\underline{\phi}}}([\mathbf{i}(k)])}
$$

for all $\mathbf{i} \in B_{\mathrm{p}}$, Theorem 4.1 implies that $\operatorname{dim} \pi\left(B_{\mathrm{p}}\right) \geq s_{\underline{\phi}} \underline{\beta}(\mathrm{p})$. If the construction is $\phi$-estimated, with estimating function $\phi(\mathbf{i})=-\log r_{i_{1}}$, then

$$
\operatorname{dim} \pi\left(B_{\mathrm{p}}\right)=\operatorname{Dim} \pi\left(B_{\mathrm{p}}\right)=s(\mathrm{p}):=\frac{\sum_{j \in M} p_{j} \log p_{j}}{\sum_{j \in M} p_{j} \log r_{j}} .
$$

Formula (5.3) was obtained in $[14,15]$ for self-similar constructions.

5.3. Measure-theoretic size of overlapping sets. The overlapping set associated with the Cantor-like construction (2.1) is defined by $\Theta:=\left\{x \in E: \operatorname{card}\left\{\pi^{-1}(x)\right\}\right.$ $>1\}$. The following theorem generalizes [14, Theorem 1], and it can be proved in a similar way.

Theorem 5.1. Let $E$ be the limit set generated by a Cantor-like construction driven by maps (see Example 3.8). Assume that the open set condition (3.7) holds.

i) Let $\Omega=\left\{\mathbf{i} \in M^{\infty}: \delta_{\mathbf{j}}(\mathbf{i})>0\right.$ for all $\left.\mathbf{j} \in M^{*}\right\}$; then $\Omega \cap \pi^{-1}(\Theta)=\emptyset$.

ii) For any $\tau$-invariant and ergodic probability measure such that $\nu([\mathbf{i}])>0$ for all $\mathbf{i} \in M^{*}, \nu\left(\pi^{-1}(\Theta)\right)=0$.

The simple construction below shows that, under the hypotheses of Theorem 5.1 , the Billingsley dimensions of the $\nu$-null set $\pi^{-1}(\Theta)$ can be arbitrarily close to one. Consider the $N$-dimensional unit cube $Q_{N}=[0,1]^{N}$ as the self-similar set generated by the set $\Psi_{N}=\left\{\varphi_{j}: j=0,1, \ldots, 2^{N}-1\right\}$, where $\varphi_{j}\left(x_{1}, x_{2}, \ldots, x_{N}\right)=$ 
$\frac{1}{2}\left(\left(x_{1}, x_{2}, \ldots, x_{N}\right)+\left(b_{1}(j), b_{2}(j), \ldots, b_{N}(j)\right)\right)$, and $\left(b_{1}(j), b_{2}(j), \ldots, b_{N}(j)\right) \in\{0,1\}^{N}$ are the first $N$ digits of the binary expansion of $j$. From (3.8) we get $s=N$. Since the overlapping set $\Theta_{N}$ is a union of $N$ hyperplanes, Lemma 3.2 implies $\operatorname{dim}_{\nu_{N}} \pi^{-1}\left(\Theta_{N}\right) \geq 1-\frac{1}{N}$, where $\nu_{N}$ is the product measure associated with the uniform probability on $\left\{0,1, \ldots, 2^{N}-1\right\}$. Although $\nu_{N}\left(\pi^{-1}\left(\Theta_{N}\right)\right)=0$ from Theorem 5.1 , the inequality above shows that the Billingsley dimensions of $\pi^{-1}\left(\Theta_{N}\right)$ can be arbitrarily close to 1 .

5.4. Two exceptional subsets of a self-similar space. We exhibit two subsets of a self-similar set $E$ which have full dimension (i.e. $\operatorname{dim} E$ ), but also have null Hausdorff and packing measures. Let

$$
\Xi=\bigcup_{\mathbf{j} \in M^{*}}\left\{\mathbf{i} \in M^{\infty}: \delta_{\mathbf{j}}(\mathbf{i})=0\right\} .
$$

Under the hypotheses of Theorem 5.1, we have that $\pi^{-1}(\Theta) \subset \Xi$, which provides a characterization of the points in the overlapping set in terms of the asymptotic frequencies of finite sequences of their associated codes.

Theorem 5.2. Let $E$ be the self-similar set generated by $\Psi=\left\{\varphi_{j}: j \in M\right\}$ which satisfies condition (3.7). Let $\nu_{s}=\chi_{1}^{\infty} \mathrm{p}_{s}$, where $\mathrm{p}_{s}=\left(r_{j}^{s}: j \in M\right)$ and $s$ is defined in (3.8). Then $\nu_{s}(\Xi)=0$, but $\operatorname{dim}_{\nu_{s}} \Xi=\operatorname{Dim}_{\nu_{s}} \Xi=1$. As a consequence $H^{s}(\pi(\Xi))=P^{s}(\pi(\Xi))=0$, but $\operatorname{dim} \pi(\Xi)=\operatorname{Dim} \pi(\Xi)=s$.

Proof. If $\Omega$ is the set in Theorem 5.1, we have $\Xi \cap \Omega=\emptyset$, and thus $\nu_{s}(\Xi)=0$. Since $\nu_{s}$ is isomorphic to the measures $\left.\left(H^{s}(E)\right)^{-1} H^{s}\right|_{E}$ and $\left.\left(P^{s}(E)\right)^{-1} P^{s}\right|_{E}[11,12]$, $H^{s}(\pi(\Xi))=P^{s}(\pi(\Xi))=0$. For $\mathbf{j} \in M^{k}$, let $A(\mathbf{j})=\left\{\mathbf{i}: \lim _{n \rightarrow+\infty} \operatorname{dist}\left(\boldsymbol{\delta}(\mathbf{i}, n), \Lambda_{\mathbf{j}}\right)=\right.$ $0\}$, where $\boldsymbol{\delta}(\mathbf{i}, n)=\left(\delta_{\mathbf{q}}(\mathbf{i}, n)\right)_{\mathbf{q} \in M^{k}}$, and

$$
\Lambda_{\mathbf{j}}=\left\{\left(\eta_{\mathrm{q}}\right)_{\mathrm{q} \in M^{k}}: \sum_{\mathrm{q} \in M^{k}} \eta_{\mathrm{q}}=1, \eta_{\mathbf{j}}=0, \eta_{\mathrm{q}}>0 \text { for } \mathrm{q} \neq \mathbf{j}\right\}
$$

Billingsley [1, Theorem 7.1] proved that

$$
\operatorname{dim}_{\nu_{s}} A(\mathbf{j})=\sup _{\left(\eta_{\mathrm{q}}\right) \in \Lambda_{\mathrm{j}}} \frac{\sum_{\mathrm{q}} \eta_{\mathrm{q}} \log \eta_{\mathrm{q}}}{\sum_{\mathrm{q}} \eta_{\mathrm{q}} \log r_{\mathrm{q}}^{s}} \leq 1 .
$$

It can be seen that the supremum in (5.5) is given by $t(\mathbf{j}) / s$, where $t=t(\mathbf{j})<s$ is defined by $\sum_{\mathrm{q} \in M^{k} \backslash\{\mathbf{j}\}} r_{\mathrm{q}}^{t}=1$. Since $\Xi=\bigcup_{\mathbf{j} \in M^{*}} A(\mathbf{j})$ and $t(\mathbf{j}) \uparrow s$ as $l(\mathbf{j}) \rightarrow+\infty$, the $\sigma$-stability of $\operatorname{dim}_{\nu_{s}}$ implies that $\operatorname{dim}_{\nu_{s}} \Xi=1$. Lemma 3.3 gives $\operatorname{dim} \pi(\Xi)=$ $\operatorname{Dim} \pi(\Xi)=s$.

For $\mathrm{p} \in \mathcal{P}^{+}$, let $B_{\mathrm{p}}^{(\infty)}=\bigcap_{\mathbf{j} \in M^{*}}\left\{\mathbf{i}: \delta_{\mathbf{j}}(\mathbf{i})=p_{j_{1}} p_{j_{2}} \ldots p_{j_{l(\mathbf{j})}}\right\}$, which we call the Besicovitch supernormal set associated with $(\pi, \mathrm{p})$. The following corollary gives the geometric size of the set of points not lying in a supernormal component of a self-similar set.

Corollary 5.3. Assume that the hypotheses of Theorem 5.2 hold. The set $E \backslash$ $\bigcup_{\mathrm{p} \in \mathcal{P}^{+}} \pi\left(B_{\mathrm{p}}^{(\infty)}\right)$ has Hausdorff and packing dimensions given by $s$, and also has null Hausdorff and packing s-dimensional measures.

Proof. Since $\pi(\Xi) \subset E \backslash \bigcup_{\mathrm{p} \in \mathcal{P}^{+}} \pi\left(B_{\mathrm{p}}^{(\infty)}\right)$, the claim on dimension is a consequence of Theorem 5.2. From the ergodic theorem, $\nu_{s}\left(B_{\mathrm{P}_{s}}^{(\infty)}\right)=1$, and thus $P^{s}\left(E \backslash \pi\left(B_{\mathrm{P}_{s}}^{(\infty)}\right)\right)=$ 
$H^{s}\left(E \backslash \pi\left(B_{\mathrm{p}_{s}}^{(\infty)}\right)\right)=0$ from the measure isomorphism referred to in the proof of Theorem 5.2.

5.5. A class of exceptional frequential sets. For $\mathrm{p} \in \mathcal{P}^{+}$, let $R_{\mathrm{p}}=\bigcap_{j \in M}\{\mathbf{i} \in$ $\left.M^{\infty}:\left|\delta_{j}(\mathbf{i}, n)-p_{j}\right|=O\left(n^{-1}\right)\right\}$, where $a_{n}=O\left(b_{n}\right)$ means that $a_{n} / b_{n}$ remains bounded as $n \rightarrow+\infty$. The law of the iterated logarithm implies that $\nu_{\mathrm{p}}\left(R_{\mathrm{p}}\right)=0$, where $\nu_{\mathrm{p}}=\chi_{1}^{\infty} \mathrm{p}$. Results in section 4 and a density argument allow us to prove

Theorem 5.4. Assume the hypotheses of Theorem 5.2; let $\mathrm{p} \in \mathcal{P}^{+}$. Then $\operatorname{dim} \pi\left(R_{\mathrm{p}}\right)$ $=\operatorname{Dim} \pi\left(R_{\mathrm{p}}\right)=s(\mathrm{p})$, but $H^{\mathrm{s}(\mathrm{p})}\left(\pi\left(R_{\mathrm{p}}\right)\right)=P^{\mathrm{s}(\mathrm{p})}\left(\pi\left(R_{\mathrm{p}}\right)\right)=0$, where $s(\mathrm{p})$ is defined in $(5.3)$.

Proof. Inequality $\operatorname{dim} \pi\left(R_{\mathrm{p}}\right) \geq s(\mathrm{p})$ follows from Theorem 4.1, since $\operatorname{dim}_{\nu_{\mathrm{p}}}\left(R_{\mathrm{p}}\right)=1$ $\left[1\right.$, Theorem 7.1], and $\lim _{k \rightarrow+\infty} \frac{\log \nu_{\mathrm{p}}([\mathbf{i}(k)])}{\log \nu_{s}([\mathbf{i}(k)])}=\frac{s(\mathrm{p})}{s}$ for all $\mathbf{i} \in R_{\mathrm{p}}$. To prove that $P^{s(\mathrm{p})}\left(\pi\left(R_{\mathrm{p}}\right)\right)=0$, define for $q \in \mathbb{N}$,

$$
R_{\mathrm{p}}(q)=\bigcap_{j \in M}\left\{\mathbf{i} \in R_{\mathrm{p}}:\left|\delta_{j}(\mathbf{i}, n)-p_{j}\right|<\frac{q}{n} \text { for all } n \text { large enough }\right\} .
$$

Let $X(j)=x_{j}:=\log p_{j}-s(\mathrm{p}) \log r_{j}$, and notice that $\sum_{j \in M} p_{j} x_{j}=0$. Writing $S_{n}^{X}(\mathbf{i}):=\sum_{k=1}^{n} X\left(i_{k}\right)$, it follows from (5.2) and (5.6) that

$$
\left|S_{n}^{X}(\mathbf{i})\right| \leq n \sum_{j \in M}\left|\delta_{j}(\mathbf{i}, n)-p_{j}\right|\left|x_{j}\right| \leq q \sum_{j \in M}\left|x_{j}\right|=: C_{q}
$$

for $\mathbf{i} \in R_{\mathrm{p}}(q)$ and for all $n$ large enough. For $t>0$ and $x \in E$, consider the density function

$$
\underline{d}_{\mathrm{p}}^{t}(x):=\sup _{\mathbf{i} \in \pi^{-1}(x)}\left\{\liminf _{k \rightarrow+\infty} \frac{\nu_{\mathrm{p}}([\mathbf{i}(k)])}{r_{\mathbf{i}(k)}^{t}}\right\} .
$$

For $x \in \pi\left(R_{\mathrm{p}}(q)\right)$, some algebra, Theorem 5.1, and inequality (5.7) give

$$
\underline{d}_{\mathrm{p}}^{\mathrm{s}(\mathrm{p})}(x) \geq \exp \left\{\liminf _{n \rightarrow+\infty} S_{n}^{X}\left(\mathbf{i}_{x}\right)\right\} \geq \exp \left(-C_{q}\right)>0,
$$

where $\mathbf{i}_{x} \in \pi^{-1}(x) \cap R_{\mathrm{p}}(q)$. Now Lemma 4.1 in [15] implies that

$$
\underline{\theta}_{\mu_{\mathrm{p}}}^{\mathrm{s}(\mathrm{p})}(x):=\liminf _{r \rightarrow 0} \frac{\mu_{\mathrm{p}}(B(x, r))}{(2 r)^{\mathrm{s}(\mathrm{p})}} \geq K \exp \left(-C_{q}\right)>0,
$$

where $\mu_{\mathrm{p}}:=\nu_{\mathrm{p}} \circ \pi^{-1}$ and $K$ is a constant which depends on $\Psi$. Since $\nu_{\mathrm{p}}\left(R_{\mathrm{p}}\right)=0$, Theorem 5.1 gives $\mu_{\mathrm{p}}\left(\pi\left(R_{\mathrm{p}}(q)\right)\right)=0$, and from $[8$, Theorem $3.16(\mathrm{a})] P^{s(\mathrm{p})}\left(\pi\left(R_{\mathrm{p}}(q)\right)\right)$ $=0$. Since $R_{\mathrm{p}}=\bigcup_{q \in \mathbb{N}} R_{\mathrm{p}}(q)$, the result follows from $\sigma$-additivity.

\section{REFERENCES}

[1] P. Billingsley, Hausdorff dimension in probability theory, Illinois J. Math. 4 (1960), 187-209. MR 24:A1750

[2] P. Billingsley, Hausdorff dimension in probability theory II, Illinois J. Math. 5 (1961), 291-298. MR 22:11094

[3] T. Bedford, Hausdorff dimension and box dimension in self-similar sets, Proc. Conf. Topology and Measure V, Binz, GDR (1987), 17-26. MR 91a:58139

[4] G. Brown, G. Michon and J. Peyriere, On the multifractal analysis of measures, J. Stat. Phys. 66 (1992), 775-790. MR 93c:58120

[5] R. Bowen, Equilibrium states and the ergodic theory of Anosov diffeomorphisms, Lecture Notes in Maths. 470, Springer-Verlag, 1975. MR 56:1364 
[6] H. CAJAR, Billingsley dimension in probability spaces, Lecture Notes in Maths. 892, Springer-Verlag, 1981. MR 84a:10055

[7] R. CAWley and R.D. Mauldin, Multifractal decomposition of Moran fractals, Adv. Math. 92 (1992), 196-236. MR 93b:58085

[8] C. CutLER, The density theorem and Hausdorff inequality for packing measures in general metric spaces, Illinois J. Math. 39 (1995), 676-694. MR 96m:28007

[9] C. DAI and S.J. TAYlor, Defining fractals in a probability space, Illinois J. Math. 38 (1994), 480-500. MR 95f:28011

[10] K.J. FALCONER, Fractal geometry- mathematical foundations and applications, Wiley, 1990. MR 92j: 28008

[11] J.E. Hutchinson, Fractals and self-similarity, Indiana Univ. Math. J. 30 (1981), 713-47. MR 82h: 49026

[12] R.D. Mauldin and M. Urbański, Infinite iterated function systems, Proc. London Math. Soc. 73 (1996), 105-154. MR 97c:28020

[13] P. Mattila, Geometry of sets and measures in euclidean spaces, Cambridge University Press, 1995. MR 96h:28006

[14] M. MorÁn and J.-M. REY, Singularity of self-similar measures with respect to Hausdorff measures, Trans. Amer. Math. Soc. 350 (1998), 2297-2310.

[15] M. Morán and J.-M. ReY, Geometry of self-similar measures, Ann. Acad. Sci. Fenn. Mathematica 22 (1997), 365-386. CMP 98:01

[16] L. Olsen, A multifractal formalism, Adv. Math. 116 (1995), 82-196. MR 97a:28006

[17] N. Patzschke, Self-conformal multifractal measures, Adv. in Applied Math. 19 (1997), 486-513.

[18] Y. Pesin and H. Weiss, On the dimension of deterministic and random Cantor-like sets, symbolic dynamics, and the Eckmann-Ruelle conjecture, preprint.

[19] Y. PEsin and H. WEISS, A multifractal analysis of equilibrium measures for conformal expanding maps and Moran-like geometric constructions, preprint.

[20] C. Tricot, Two definitions of fractional dimension, Math. Proc. Cambridge Phil. Soc. 91 (1982), 57-74. MR 84d:28013

Centre for Nonlinear Dynamics and its Applications, University College London, Gower Street, London WC1E 6BT, United Kingdom

Current address: Departamento de Análisis Económico, Universidad Complutense, Campus de Somosaguas, 28223 Madrid, Spain

E-mail address: ececo07@sis.ucm.es 